\title{
Failure of spermatogenesis in mouse lines deficient in the $\mathrm{Na}^{+}-\mathrm{K}^{+}-\mathbf{2} \mathrm{Cl}^{-}$cotransporter
}

\author{
Amy J. Pace, ${ }^{1,2}$ Eddie Lee, ${ }^{2}$ Krairek Athirakul, ${ }^{3}$ Thomas M. Coffman, ${ }^{3}$ \\ Deborah A. O'Brien, ${ }^{1,4}$ and Beverly H. Koller ${ }^{2}$
}

${ }^{1}$ Curriculum in Genetics and Molecular Biology, and

${ }^{2}$ Department of Medicine, University of North Carolina-Chapel Hill, Chapel Hill, North Carolina 27599, USA

${ }^{3}$ Department of Medicine, Duke University and Durham Veterans Affairs Medical Centers, Durham, North Carolina 27705, USA

${ }^{4}$ Department of Cell Biology and Anatomy, University of North Carolina-Chapel Hill, Chapel Hill, North Carolina 27599, USA

Address correspondence to: Beverly H. Koller, Department of Medicine, University of North Carolina-Chapel Hill, Chapel Hill, North Carolina 27599-3360, USA. Phone: (919) 962-2159; Fax: (919) 966-7524; E-mail: treawouns@aol.com.

Amy J. Pace and Eddie Lee contributed equally to this work.

Received for publication September 27, 1999, and accepted in revised form January 6, 2000.

The $\mathrm{Na}^{+}-\mathrm{K}^{+}-2 \mathrm{Cl}^{-}$cotransporter $\left(\mathrm{NKCC} 1\right.$ ) carries 1 molecule of $\mathrm{Na}^{+}$and $\mathrm{K}^{+}$along with 2 molecules of $\mathrm{Cl}^{-}$across the cell membrane. It is expressed in a broad spectrum of tissues and has been implicated in cell volume regulation and in ion transport by secretory epithelial tissue. However, the specific contribution of NKCC1 to the physiology of the various organ systems is largely undefined. We have generated mouse lines carrying either of 2 mutant alleles of the Slc12a2 gene, which encodes this cotransporter: a null allele and a mutation that results in deletion of 72 amino acids of the cytoplasmic domain. Both NKCC1-deficient mouse lines show behavioral abnormalities characteristic of mice with inner ear defects. Male NKCC1-deficient mice are infertile because of defective spermatogenesis, as shown by the absence of spermatozoa in histological sections of their epididymides and the small number of spermatids in their testes. Consistent with this observation, we show that Slc12a2 is expressed in Sertoli cells, pachytene spermatocytes, and round spermatids isolated from wild-type animals. Our results indicate a critical role for NKCC1-mediated ion transport in spermatogenesis and suggest that the cytoplasmic domain of NKCC1 is essential in the normal functioning of this protein.

J. Clin. Invest. 105:441-450 (2000).

\section{Introduction}

$\mathrm{Na}^{+}-\mathrm{K}^{+}-2 \mathrm{Cl}^{-}$cotransporters couple the electroneutral cellular uptake of 1 molecule of $\mathrm{Na}^{+}$and $\mathrm{K}^{+}$and 2 molecules of $\mathrm{Cl}^{-}$across the cell membrane. These systems are present in a broad spectrum of eukaryotic cells and are believed to play a central role in vectorial salt transport across epithelium, as well as volume regulation in epithelial and nonepithelial cells (1). Under normal physiological conditions, $\mathrm{Na}^{+}-\mathrm{K}^{+}-2 \mathrm{Cl}^{-}$cotransporters mediate a net influx of ions into cells because of the inwardly directed chemical gradients for $\mathrm{Na}^{+}$and $\mathrm{Cl}^{-}$ions (2). Molecular studies have identified 2 isoforms, the $\mathrm{Na}^{+}-\mathrm{K}^{+}-2 \mathrm{Cl}^{-}$ cotransporters NKCC1 and NKCC2, that share about $60 \%$ identity and are sensitive to loop diuretics such as furosemide and bumetanide (3). Despite these similarities, the 2 cotransporters have very distinctive patterns of expression. To date, expression of the absorptive isoform, NKCC2, has been detected only in the kidney where its expression is limited to the epithelial cells of the thick ascending limb of Henle (4). NKCC1 is also expressed in the kidney, primarily in the epithelial cells in the medullary collecting duct and in the glomerulus (5-7). In addition to the kidney, expression of this isoform can be detected in a broad spectrum of tissues where it is thought to play a critical role in fluid secretion and cell volume regulation (2).
Evidence supporting a role for NKCC1 in volume regulation and in the function of various secretory epithelia is based largely on studies that examine the impact of the loop diuretics bumetanide or furosemide on these processes $(8,9)$. In epithelia that absorb or secrete $\mathrm{Cl}^{-}-$ rich fluids, inward $\mathrm{Na}^{+}-\mathrm{K}^{+}-2 \mathrm{Cl}^{-}$cotransport often represents the first step in the movement of $\mathrm{Cl}^{-}$across the polarized cells (10). Secretion of $\mathrm{Cl}^{-}$depends on the coordinated efflux of $\mathrm{Cl}^{-}$through apical $\mathrm{Cl}^{-}$channels such as CFTR. $\mathrm{Na}^{+}-\mathrm{K}^{+}-2 \mathrm{Cl}^{-}$cotransporter activity is also believed to be important in the secretory function of epithelial tissues that produce extracellular fluid rich in $\mathrm{K}^{+}$. Presumably, $\mathrm{K}^{+}$enters these cells by active uptake through the cotransporter and the $\mathrm{Na}^{+}, \mathrm{K}^{+}$pump, and then exits across the luminal membrane of the cells through $\mathrm{K}^{+}$channels $(11,12)$. High levels of cotransporter expression have been reported in the salivary gland and the marginal cells of the inner ear, tissues that are known to produce $\mathrm{K}^{+}$-rich fluids (13-15). Expression of NKCC1 can also be detected in nonepithelial cells such as red blood cells and lymphocytes, where it is believed to be important in volume regulation in response to various physiological stimuli $(16,17)$.

Northern analysis indicates that NKCC1 is expressed in various organs of the male and female reproductive systems (18), and although it seems likely that the cotrans- 
porter is involved in fluid secretion by epithelial cells in these organs, no direct evidence assessing its contribution to the fertility of the organism has been carried out.

The testis is composed of loops of convoluted seminiferous tubules lined with epithelium containing Sertoli cells and spermatogenic cells at various stages of differentiation. The seminiferous tubule fluid that establishes the microenvironment for spermatogenesis and transports spermatozoa from the seminiferous tubules to the head of the epididymis is believed to be produced by the Sertoli cells. The composition of seminiferous tubule fluid is distinct from that of blood plasma, with 10 times higher $\mathrm{K}^{+}$and lower $\mathrm{Na}^{+}$concentrations (19). The production of a fluid with a unique ionic composition by the epithelium of the testes has led to the hypothesis that it plays a vital role in normal germ cell development or Sertoli cell function. The pathways involved in production of this fluid have not been defined, and there is no evidence to date supporting a role for NKCC1 in this process.

Throughout spermatogenesis, the germ cells are enveloped by cytoplasmic processes of the Sertoli cell. In the first phase of spermatogenesis, which occurs near the basal lamina, spermatogonia undergo a defined number of divisions and give rise to meiotic cells, the spermatocytes. As differentiation proceeds, spermatogenic cells move toward the seminiferous tubule lumen. Completion of meiosis leads to the formation of haploid spermatids that undergo a series of dramatic morphological transformations, including acrosome formation, polarization and elongation of the nuclei, condensation of the chromatin, flagellum formation, and elimination of excess cytoplasm. Spermatozoa are released from the apical surface of the Sertoli cell into the seminiferous tubule lumen. Whereas the extensive morphological changes observed during spermatogenesis suggest that adequate regulation of intracellular ions might be a critical component of this differentiation process, there is no direct evidence to date supporting a role of NKCC 1 in this process.

To address the role of NKCC 1 in the development and physiology of various epithelia, we generated 2 mouse lines carrying mutations in the Sla 12 a 2 gene. We show that whereas loss of cotransporter function does not result in severe lesions in most organ systems, even those in which high levels of expression have been demonstrated, NKCC1 function is essential for normal spermatogenesis.

\section{Methods}

Generation of NKCC1-deficient mouse lines. A genomic clone containing a $3^{\prime}$ region of the Slc12a2 gene was isolated from a FixII SV129 $\lambda$ phage library using a cDNA probe corresponding to bp 2507 to 3636 of the published mouse Slc12a2 sequence (18) and subcloned into pBluescript (Stratagene, La Jolla, California, USA), pBS/NKCC3a. DNA fragments from this genomic clone were used to prepare a Slc12a2-targeting plasmid. A 1.7-kb BamHIEcoRI region located immediately $5^{\prime}$ of exon 24 was cloned just $5^{\prime}$ of the $3^{\prime}$ end of the neomycin gene in JNS2 (20). A second 11-kb fragment extending from the Bam HI site just $3^{\prime}$ of exon 25 to the end of the cloned segment of Slc12a2 DNA was subcloned $5^{\prime}$ of the neomycin gene. A second genomic clone containing exons encoding the transmembrane domains of the Slc12a2 gene was isolated using a cDNA probe corresponding to bp 1239-2449 of the published mouse Slc12a2 sequence (18). DNA fragments from this clone were used to construct the Slc12a $2^{4506-621}$ targeting plasmid that is designed to replace a 344-bp region containing exons 9,10 , and 11 with the neomycin gene. A $5.1-\mathrm{kb}$ XbaI fragment located $5^{\prime}$ to exon 9 was cloned just $5^{\prime}$ of the $3^{\prime}$ end of the neo gene in JNS2 (20). A second fragment of 7.1-kb beginning just $3^{\prime}$ to exon 11 and extending to the NotI site $3^{\prime}$ of exon 15 of the Slc12a2 gene was subcloned just $5^{\prime}$ of the neo gene. Plasmids were linearized and introduced into the embryonic stem (ES) cell line E14TG2a and transformants were isolated as described previously (21). Cells were screened for targeted integration of the plasmid by Southern blot analysis as described below, and targeted cell lines were microinjected into 3.5-day C57BL/6J blastocysts to generate chimeric animals. These were subsequently mated to B6D2 (C57BL/6J $\times$ DBA/2J F1) animals to generate animals heterozygous for the mutant allele.

Genotyping. DNA was isolated from ES cell lines and from tail biopsies, as described previously $(21,22)$, and the genotype was determined by Southern blot analysis. To detect targeted introduction of the Slc12a241065-1137 plasmid, DNA was digested with $\mathrm{XbaI}$ and probed with an 800-bp Bam HI fragment (Figure 1a). To detect targeted introduction of the Slc12a $2^{4506-621}$ plasmid, DNA was digested with HindIII and probed with a $1.8 \mathrm{-kb}$ fragment (Figure 1a).

Northern analysis. Populations of Pachytene spermatocytes and round spermatids with more than $90 \%$ purity were isolated from the testes of adult mice and Sertoli cells from 17-day-old mice as described previously (23, 24). Total RNA was isolated from cells and tissues by phenol/chloroform extraction using RNAzol B (Tel-Test, Inc., Friendswood, Texas, USA) and 10-20 $\mu \mathrm{g}$ was electrophoresed on a $1.1 \%$ formaldehyde, $1.2 \%$ agarose denaturing gel in the presence of ethidium, as described previously (25). After transfer to Immobilon-NC nitrocellulose membrane (Millipore Corp., Bedford, Massachusetts, USA), filters were hybridized with radiolabeled DNA probes in Quick-Hybe (Stratagene) for 1 hour at $68^{\circ} \mathrm{C}$.

RT-PCR analysis of Slc12a2 RNA present in Slc12a $2^{\Delta 1065-1137-}$ homozygous animals. RT-PCR generated cDNA (Invitrogen Corp., Carlsbad, California, USA), which was amplified using the primers NKCC3a, 5'-CAG GGC CTG CTT TAC TTC ATC TTG-3'; and NKCC3b, 5'GCC TTT CCG TGC GAC TGG-3'. PCR products were analyzed on $2 \%$ agarose gels.

Histological analysis and transmission electron microscopy. Reproductive organs were fixed in Bouin's solution, embedded in paraffin, sectioned at $8 \mu \mathrm{m}$, and stained with hematoxylin and eosin $(\mathrm{H} \& \mathrm{E})$ or hematoxylin and 


\section{Figure 1}

Generation of mouse lines with mutations in the $S / c 12 a 2$ gene. (a) Restriction maps of the endogenous S/c12a2 locus, the S/c12a2 targeting constructs, and S/c12a2 locus after homologous recombination with the targeting plasmids. Upon homologous recombination, the $S / c 12 a 2^{\Delta 1065-1137}$ construct replaces a 217-bp region of the S/c12a2 gene, which includes exons 24 and 25, with the selectable marker gene neomycin (neo). Relevant restriction sites are abbreviated as follows: B, BamHI; P, Pvul; R, EcoRI; X, Xhol. The $S / c 12 a 2^{-1-}$ construct is designed, upon homologous recombination, to replace a 344-bp region of the gene encompassing exons 9,10 , and 11 with the neo gene. Relevant restriction sites are abbreviated as follows: B, BamHI; H, HindIII; N, Notl; X, Xbal. The probes used to detect homologous recombination events by Southern blot analysis are indicated. (b) Model of the $\mathrm{Na}^{+}-\mathrm{K}^{+}-$ $2 \mathrm{Cl}^{-}$cotransporter with deleted regions indicated. Circles represent amino acid residues. The amino acids encoded by the regions

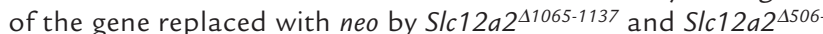
621 are indicated by filled circles and shaded circles, respectively. (c) Southern blot analyses of offspring generated by the intercross of mice heterozygous for each of the S/c12a2 mutant alleles. An 800-bp BamHI genomic fragment detects the introduction of an $X b a l$ site after targeted integration of the $S / c 12 a 2^{\Delta 1065-1137}$ plasmid (left panel). For S/c12a2 ${ }^{\Delta 506-621}$ (right panel), a 1.8-kb genomic fragment that hybridizes upstream of the targeted region was used as a probe to detect the change in Hindlll restriction fragment length that occurs with proper integration of the targeting construct. (d) Northern analysis of NKCC1 expression in salivary

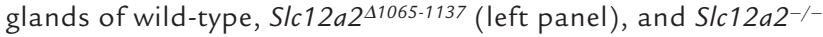
(right panel) heterozygous and homozygous animals. Abundant NKCC1 RNA is observed in mice homozygous and heterozygous for either mutation. A slightly smaller NKCC1 transcript resulting from the deletion of exons 23 and 24 is seen in RNA prepared from $S / c 12 a 2^{\Delta 1065-1137}$ mice, whereas a novel transcript is present in the samples from the $S / c 12 a 2^{-/-}$mice. Analysis with an actinspecific probe indicates equal RNA sample loading. (e) Analysis of the NKCC1 mRNA remaining in the S/c12a $2^{\Delta 1065-1137}$ animals by RT-PCR. RT-PCR of RNA from wild-type animals yields a fragment of approximately $1100 \mathrm{bp}$, whereas a fragment of approximately 900 bp was amplified from the heterozygous and homozygous mutant cDNAs, consistent with loss of 195-bp of coding sequence in this mutant line. (f) Analysis of the novel RNA tran-

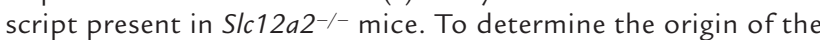
novel transcript seen in $\mathbf{d}$, the Northern blot was analyzed with a probe specific for the neomycin gene. A band identical in size to that observed using the NKCC1-specific probe is observed in RNA obtained from S/c12a2-/- mice.

periodic acid-Schiff (H\&PAS). For transmission electron microscopy, mice were deeply anesthetized and pericardially infused with $4 \%$ paraformaldehyde. Onemillimeter sections of the testes were postfixed in $1 \%$ osmium tetraoxide, dehydrated in a graded series of ethanol washes, and embedded in a resin mixture consisting of 1 part complete Spurr's resin and 1 part complete Poly-bed 812 resin (Polysciences Inc., Warrington, Pennsylvania, USA). Seventy-nanometer-thin sections were cut with a diamond knife, mounted in a 200-mesh grid, and examined with a Zeiss EM 910 (LEO Electron Microscopy Inc., Thornwood, New York, USA) transmission electron microscope. a

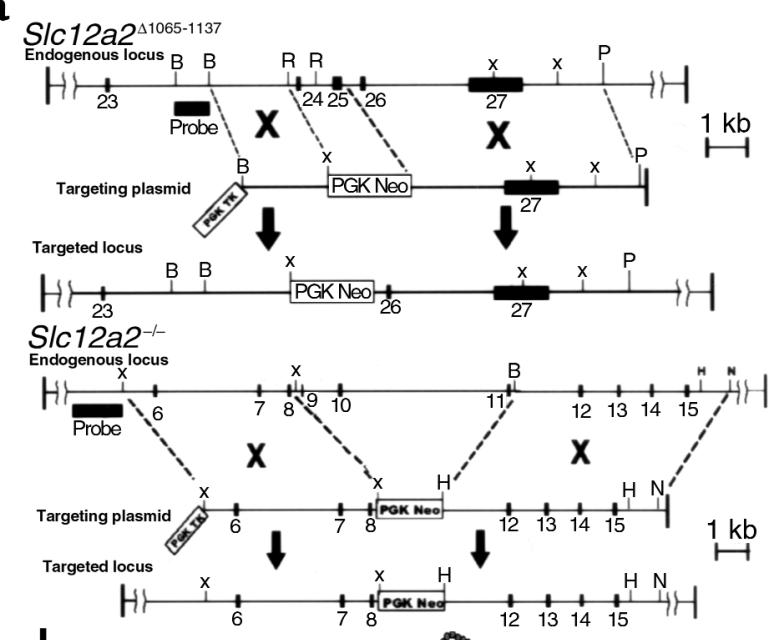

b

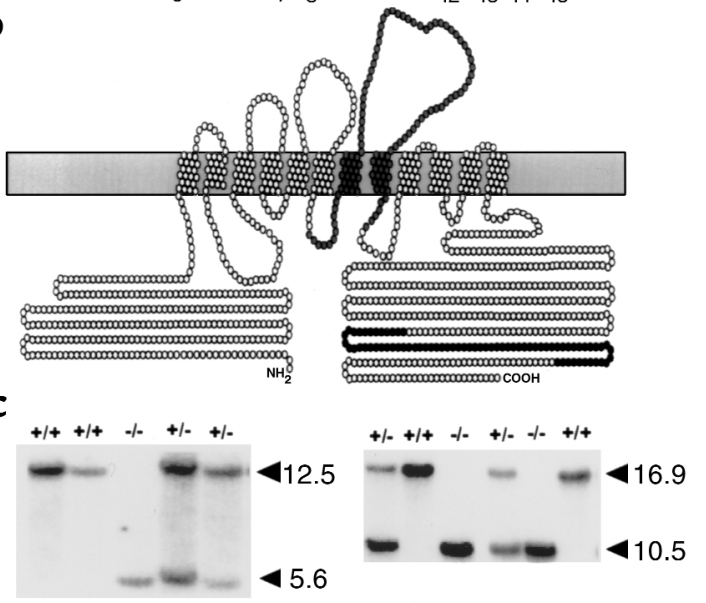

d

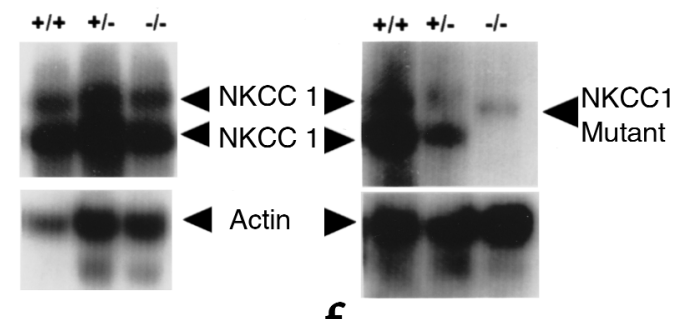

e

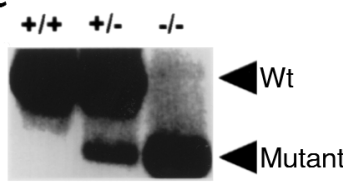

f

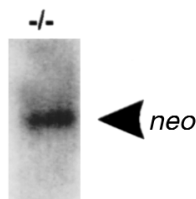

Motion and stereotypy studies. For each study, 5 sets of age- and sex-matched Slc12a2 $2^{\Delta 1065-1137}$ mice, shaker-2 mice, and wild-type controls were monitored under standardized environmental conditions using an automated TruScan Multi-Parameter Activity Monitor (Coulbourn Instruments, Allentown, Pennsylvania, USA), in a $671-\mathrm{cm}^{2}$ Plexiglas chamber with beam spacing of $1.52 \mathrm{~cm}$. Ambulatory distance, measured as the sum total of all vectored $X-Y$ coordinate changes, less the vectored distance in stereotypic movements of each mouse, was recorded in 5-minute intervals over a 2hour period, and the raw numbers were averaged to give values for each 5 -minute interval. Values represent 


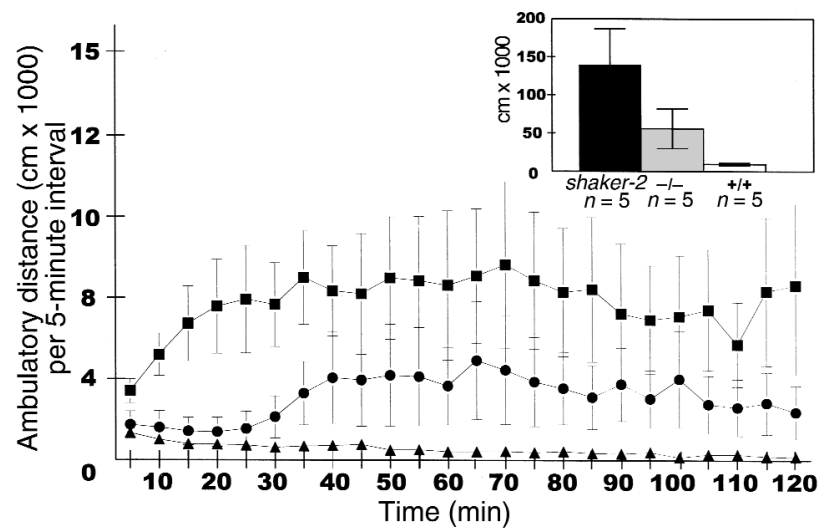

Figure 2

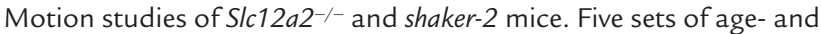
sex-matched S/c12a2---, shaker-2, and wild-type control mice were tested for 4-hour periods using a TruScan Multi-Parameter Activity Monitor. Ambulatory distance is measured as distance traveled minus stereotypic movements. Filled circles represent the average ambulatory distance per 5-minute interval for the S/c12a2-/- mice, with error bars indicated. Filled triangles represent the wild-type controls, with error bars indicated, and filled squares represent the shaker-2 mice, with error bars indicated. Inset: Bar graph of total ambulatory distance for the $S / c 12 a 2^{-/-}$mice versus the shaker-2 mice versus wild-type controls, with error bars indicated.

means \pm SEM and statistical significance was analyzed by 2 -tailed $t$ test on 2 -hour summed data.

Systolic blood pressure measurements in conscious mice. Systolic blood pressures were measured in conscious mice using a computerized tail-cuff system (Visitech Systems, Carey, North Carolina, USA) that determines systolic blood pressure using a photoelectric sensor (26, 27). To determine the effects of altered dietary sodium on blood pressure, wild-type $(n=5)$ and Slc12a2 $11065-1137$ $(n=5)$ mice were first fed a control diet containing $0.4 \%$ sodium chloride for 2 weeks. This was followed by a 14day period in which the animals were given a high-salt diet containing $6 \%$ sodium chloride. Following the high-salt feeding, the mice were re-equilibrated on the control diet for 7 days. The animals were then fed a lowsalt diet containing less than $0.02 \%$ sodium chloride for the next 14 days. All diets were purchased from HarlanTeklad Laboratory (Madison, Wisconsin, USA). Systolic blood pressures were measured at least 5 times per week throughout the period of study.

Urine osmolality measurements before and after desmopressin administration. We examined the effect of desmopressin (dDAVP; Rhone-Poulenc Rorer, Collegeville, Pennsylvania, USA) on urine osmolality in wild-type ( $n$

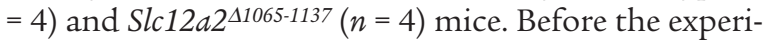
ments, animals were allowed free access to drinking water and $0.4 \% \mathrm{NaCl}$ chow. Following the collection of a baseline urine sample by bladder massage, mice were injected with $1.0 \mu \mathrm{g} / \mathrm{kg}$ desmopressin subcutaneously, and water bottles were removed. Urine samples were collected 4 hours after injections and urine omolalities were immediately measured using a vapor pressure osmometer (Wescor Inc., Logan, Utah, USA).

\section{Results}

Generation of NKCC1-deficient mice. The Slc12a2 gene was disrupted in the ES cell line using the 2 targeting plasmids depicted in Figure 1a. Primary amino acid sequence analysis of the $\mathrm{Na}^{+}-\mathrm{K}^{+}-2 \mathrm{Cl}^{-}$cotransporter, including hydrophobicity analysis and comparisons of the sequence of NKCC1 to other cotransporters, has led to the division of this protein into 3 structural regions. The $\mathrm{NH}_{2}$ - and $\mathrm{COOH}$-terminal regions contain residues that are believed to be sites of regulatory phosphorylation. Unlike the $\mathrm{NH}_{2}$-terminal region, the $\mathrm{COOH}$ terminus shows a high level of conservation between species. A 500 -amino acid central region of the protein is predicted to contain 12 transmembrane helices $(18,28)$. The first targeting plasmid, pSlc12a2 $2^{\Delta 1065-1137}$, was designed to remove a 217-bp region of the Slc12a2 gene containing exons 24 and 25 . These exons encode the portion of the 3 ' cytoplasmic domain of the protein indicated in Figure 1b. The second plasmid, pSlc12a2 $2^{\Delta 506-621}$, was designed to remove a 344-bp region of the Slc12a2 gene that contains exons 9, 10, and 11 . These exons encode transmembrane domains 7 and 8 of the NKCC1 protein.

Targeted ES cells were used to generate mice heterozygous for each of the mutated Slc12a2 alleles. Heterozygous animals were intercrossed and the frequency of offspring homozygous for the mutations present in the litters was determined (Figure 1c). In each case animals homozygous for the mutant allele were present in the litters at the expected frequency. To determine whether the introduction of the targeting plasmid resulted in loss of NKCC1 expression, Northern blot analysis of salivary gland RNA was carried out. Analysis of RNA from animals homozygous for the Slc12a $2^{41065-1137}$ mutation (Figure 1d, left), indicates levels of expression similar to those observed in the control animals. A small shift in size of the NKCC1 mRNA prepared from the Slc12a2 $2^{\Delta 1065-1137}$ animals, consistent with the deletion of exons 23 and 24 of the Slc12a2 gene, is seen. To determine if the homologous recombination event introduced the expected mutation, mRNA prepared from the Slc12a2 $2^{\Delta 1065-1137}$ animals was subjected to RT-PCR using primers flanking the region predicted to be lost during the recombination event. These primers are expected to amplify an 1100-bp fragment from wild-type NKCC1 cDNA and a 900-bp fragment from cDNA generated from mRNA derived from the Slc12a $2^{\Delta 1065-1137}$ mutant allele. Figure 1e indicates that, as expected, only normal NKCC1 transcript is present in mRNA prepared from tissue obtained from wild-type mice. In contrast, both mutant and native NKCC1 mRNA are present in RNA prepared from tissue obtained from heterozygous animals. Finally, only the mutated 900-bp fragment is amplified when RNA obtained from homozygous Slc12a $2^{\Delta 1065-1137}$ animals is used as a substrate. This mRNA is expected to result in the expression of an NKCC1 protein with a shortened cytoplasmic tail as a result of the absence of amino acids 1065 to 1137 . We therefore refer to this mutant as a deletion mutation and not a null allele in this report. 


\section{Figure 3}

Histological examination of testes from 3-4-day old and 21-day-old wild-type (a, b) and S/c12a2-/- $(\mathbf{c}, \mathbf{d})$ mice. Analysis of testes from 3-4-day-old mice revealed no difference between the Slc12a2-/- (c) and wild-type males (a). a and $\mathbf{c}$, bars, $50 \mu \mathrm{m}$. Analysis of testes from 21-day-old mice showed a difference in the timing of the formation

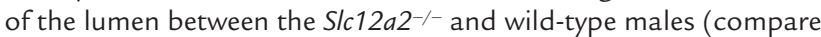
b to d). Seminiferous tubules in wild-type testes have well-developed lumens, whereas lumen formation appears to be delayed in S/c12a2-/testes, and the central region of the tubules still contains many germ cells. $\mathbf{b}$ and d, bars, $100 \mu \mathrm{m}$.

No wild-type NKCC1 RNA is detected on Northern analysis of RNA prepared from the salivary glands of mice homozygous for the Slc12a $2^{\Delta 506-621}$ mutation. However, low levels of a novel transcript that also hybridizes to a neomycin-specific probe are observed. Because this is unlikely to generate a functional protein, the mutation introduced by pSlc12a $2^{4506-621}$ has presumably generated a null Slc12a2 allele and will be referred to as $\operatorname{Slc} 12 a 2^{-/-}$throughout this report.

Survival, growth, and health of the NKCC1-deficient mice.

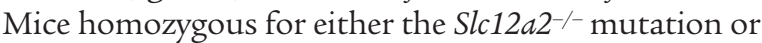
the Slc12a $2^{41065-1137}$ mutation could not be identified at birth by observation alone. However, within 2 weeks the NKCC1-deficient pups could be distinguished from littermates because of their smaller size. Pups from 8-10 litters generated from heterozygous intercrosses of each mutant line were weighed and genotyped 14 days after birth. Pups homozygous for both of the Slc12a2 muta-
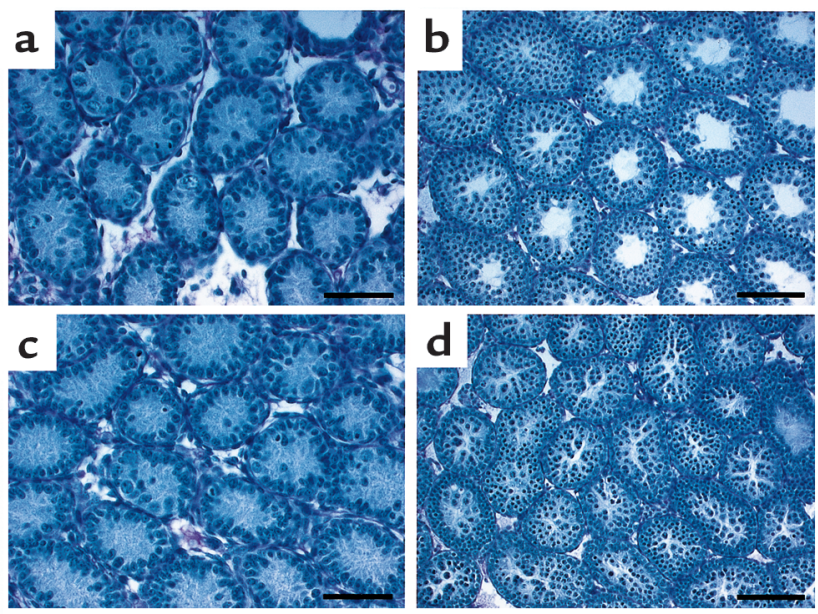

tions had weights that were, on average, only $55 \%$ of that of wild-type littermates (Student's $t$ test: Slc12a2 ${ }^{41065-1137}$ to wild-type, $P<4.2 \times 10^{-8}$; Slc12a2-/- to wild-type, $P<4.4$ $\left.\times 10^{-8}\right)$. No significant difference was observed between the wild-type and heterozygotes of each respective group, and there was no significant difference between the 2 mutations, suggesting that the null mutation and the deletion mutation have a similar impact on the growth of the pups. In both lines the difference in size becomes less pronounced as the mice reach maturity.

In addition to the smaller size, NKCC1-deficient mice can also be distinguished based on their ataxic gait. By 3 weeks of age these mice display increased motor and
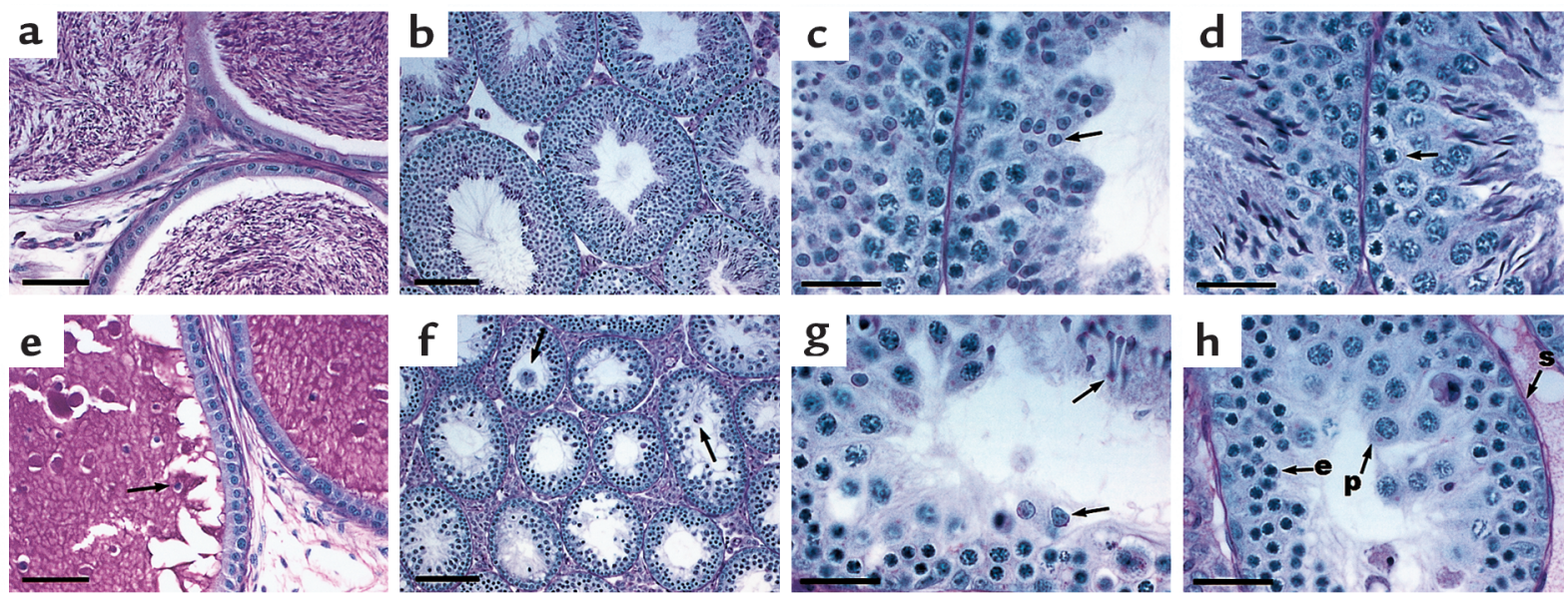

Figure 4

Histological analysis of the reproductive tract of wild type (a-d) and NKCC1-deficient (e-h) animals. Representative sections are from S/c12a2-/animals, although the defect in both NKCC1-deficient lines is identical. Sections through the cauda epididymis of both normal (a) and $S / c 12 a 2^{-/-}$(e) mice show columnar epithelium with stereocilia on the apical surface. In wild-type mice the epididymal lumen is filled with mature sperm. In contrast, no sperm are observed in NKCC1-deficient animals, and the epididymal lumen is filled with PAS-staining material and

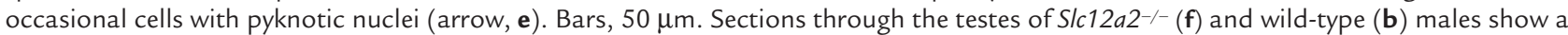
decrease in the size of the seminiferous tubules, resulting from a variable loss of germ cells from the seminiferous epithelium. Multinucleated cells are often seen in the lumens of the seminiferous tubules of the NKCC1-deficient males (arrows, $\mathbf{f}$ ). Bars, $100 \mu \mathrm{m}$. High-magnification photomicrographs of the seminiferous tubules of normal animals $(\mathbf{c}, \mathbf{d})$ show tubules containing developing germ cells at all stages of spermatogenesis. This includes early spermatocytes (arrow, $\mathbf{d}$ ), elongating spermatids (arrow, c), and later condensed spermatids (d) near the

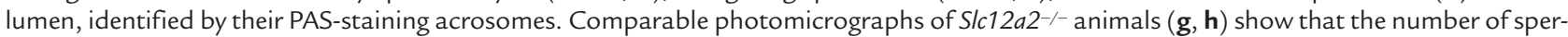
matids is substantially reduced. A small number of developing spermatids with PAS-stained acrosomes can be identified (arrows, $\mathbf{g}$ ). Normal Sertoli cells were observed ( $\mathrm{s}$ in $\mathbf{h}$ ) along the basement membrane of the seminiferous epithelium, as well as spermatogonia and/or early spermatocytes ( $\mathrm{e}$ in $\mathbf{h}$ ) and a reduced number of large pachytene spermatocytes ( $\mathrm{p}$ in $\mathbf{h}$ ). In $\mathbf{c}, \mathbf{d}, \mathbf{g}$, and $\mathbf{h}$, bars, $30 \mu \mathrm{m}$. 
stereotypic activity, characterized by periods of circling in a clockwise direction and head tossing. Motor coordination, balance, and ataxia in mice can be tested and quantified using a Rotorod test (29). Even after extensive training on the apparatus, the NKCC1-deficient mice failed to remain on the rod for more than 30 seconds, whereas the majority of the wild-type mice could complete a 3-minute session (data not shown). In addition, NKCC1-deficient mice failed to startle in response to noise. The increased motor activity, head tossing, and poor balance combined with the hearing deficit observed in the mutant mice is similar to that reported in a number of mouse lines with mutations that affect the inner ear (30-32). The hearing deficit and altered motor activity is consistent with the demonstration of high levels of NKCC 1 in the inner ear (14) and has also been reported recently by other investigators who have introduced mutations into Slc12a2 (33). It is also observed in a mouse line in which a spontaneous mutation occurred in this gene (34). However, NKCC1 is also expressed in virtually all regions of the brain, including regions such as the cerebellum, that are involved in regulation of motor activity (35). To determine whether alterations in the inner ear can account for all of the behavioral changes seen in the mutant animals, we compared the NKCC1-deficient mice directly to shaker-2 (sh-2) mice. As a result of a mutation in the Myo-15 gene that encodes an unconventional myosin (30), these mice are deaf and suffer from an associated vestibular defect that leads to circling behavior and increased stereotypic behavior such as head tossing. Age-matched shaker-2 and NKCC1-deficient mice were of similar size at 8 weeks of age, and both were slightly smaller in size than wild-type mice. Wild-type, NKCC1-deficient, and shaker-2 mice were subjected to an open-field locomotion test, and total ambulatory distance traveled was measured (Figure 2). Ambulatory distance measured over 2 hours was increased dramatically in both the shaker-2 and the Slc12a2 $2^{-/}$mice. The majority of this increase in motor activity was the result of circling behavior, primarily in a clockwise direction, present in both lines. Stereotypic activity, which includes the head tossing characteristic of the shaker-2 mice, was similar in the 2 lines during periods of rest (data not shown). Thus, direct comparison of the NKCC1-deficient mice to shaker-2 mice suggests that a lesion in the inner ear alone can account for all of the observed changes in motor and stereotypic activity in the NKCC1-deficient mice.

NKCC1 has a broad tissue distribution; however, no histological changes were noted in the kidney, salivary glands, or lungs of the NKCC1-deficient mice, even at advanced age. In addition, analysis of serum electrolytes of wild-type and Slc12a $2^{41065-1137}$ animals failed to reveal differences between the 2 groups of animals. White blood cell differentials, hematocrits, and platelet counts were also normal in NKCC1-deficient animals (data not shown).

Examination of renal function and physiological parameters in NKCC1-deficient animals. NKCC1 is 1 of 3 transporters found in the kidney and is expressed within the glomerulus, the collecting duct, and the glomerular afferent arteriole (7). Accordingly, it has been suggested that this transporter may play a role in regulating blood pressure and salt balance. To examine the actions of NKCC 1 in blood pressure regulation, we measured systolic blood pressure in wild-type and NKCC1 $1^{\Delta 1065-1137}$ mice. Systolic blood pressures were slightly lower in the Slc12a $2^{41065-1137}$ mice $(100 \pm$ $7 \mathrm{mmHg}$ ) than in controls $(0.4 \%$ sodium content diet; 108 $\pm 8 \mathrm{mmHg}$ ), but this difference did not achieve statistical significance $(P=0.54)$. Varying dietary sodium content from $6 \%$ to less than $0.02 \% \mathrm{NaCl}$ did not significantly alter systolic blood pressures in either the NKCC1-deficient or wild-type groups (data not shown). Thus, NKCC1 does not play an essential role in blood pressure regulation or in adaptation to altered dietary sodium intake. In the kidney, NKCC1 is also highly expressed in the inner medulla (7), where it might contribute to the development and/or maintenance of the medullary osmolar gradient. Because this osmolar gradient is critical for urinary concentrating ability, we compared urine osmolalities in Slc12a2 $2^{4065-1137}$ and wild-type mice. Baseline urine osmolalities were sim-
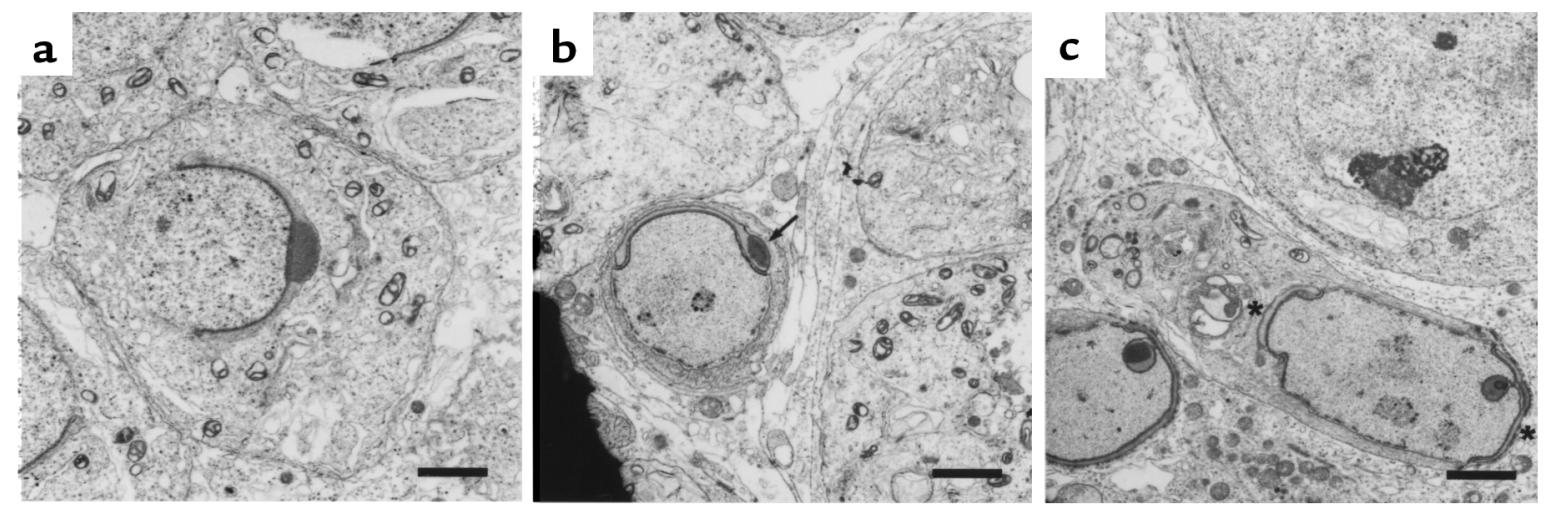

Figure 5

Analysis of acrosomal development in NKCC1-deficient mice by electron microscopy. (a) An electron micrograph of a wild-type spermatid showing a central acrosomal granule within a cap-phase acrosomal vesicle. Developing spermatids of NKCC1-deficient mice (b, c) have abnormal structures. Examples include abnormal placement of the acrosomal granule (arrow, b) and a dual vesicular structure with 2 acrosomes at opposite poles of one nucleus (* in c). Bars, $2.42 \mu \mathrm{m}$. 


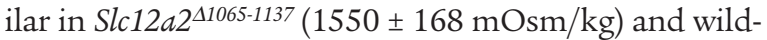
type mice $(1823 \pm 276 \mathrm{mOsm} / \mathrm{kg} ; P=0.51)$ given free access to drinking water. To determine their capacity for urinary concentration, we measured urinary osmolality after administration of a pharmacological dose $(1 \mathrm{mg} / \mathrm{kg})$ of the vasopressin analogue dDAVP. Administration of dDAVP caused significant increases in urine osmolalities in both groups, and there was no significant difference in

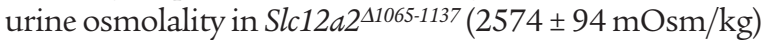
and wild-type mice $(2816 \pm 276 \mathrm{mOsm} / \mathrm{kg} ; P=0.63)$ after vasopressin. These data indicate that the absence of NKCC1 does not significantly alter urinary concentrating functions in the kidney.

NKCC1-deficient males are infertile. No histological changes were noted in the ovary, oviduct, or uterus of the NKCC1-deficient animals despite high levels of expression of the Slc12a2 gene in these tissues (data not shown). Furthermore, female NKCC1-deficient mice are fertile although, in general, they become pregnant less frequently than heterozygous or wild-type littermates. However, litters born to the NKCC1-deficient females rarely survive. We attribute this, at least in part, to the abnormal motor activity of these animals.

In contrast, NKCC1-deficient males of both lines housed with wild-type females failed to produce litters even after extended breeding. Specifically, 5 Slc12a2-/males co-housed with females for 15 weeks after reaching sexual maturity failed to impregnate females. Similarly, 8 Slc12a2 $2^{-/-}$males co-housed with females for more than 5 weeks did not impregnate the animals. When presented with ovulating females, 2 out of 6 Slc12a2-/- males mated, as evidenced by the presence of copulatory plugs. The altered motor activity of these animals may contribute to these lower mating frequencies. The weights of the seminal vesicles of 7 Slc12 $\mathrm{a}^{-/-}$animals were similar to the weights of this gland in wild-type males, suggesting that testosterone levels were normal in the mutant mice.

Histological examination of testes from one 3-4-dayold Slc12a2 $2^{\Delta 1065-1137}$ male and a wild-type littermate revealed no difference in seminiferous tubule maturation between these 2 animals (Figure $3 a$ and $3 c$ ). In contrast, examination of testes from four 21-day-old Slc12 $\mathrm{a}^{-/-}$males and wild-type littermates revealed that the NKCC1-deficient males appear to have a defect in the timing of lumen formation. In wild-type testes the lumen has been formed in a majority of the seminiferous tubules (Figure 3b), whereas in NKCC1-deficient males the lumen has not yet formed and germ cells still appear to occupy the central region of the tubules (Figure 3d). We next examined the reproductive organs of mature wild-type and NKCC1-deficient males by histological analysis. Studies included examination of reproductive

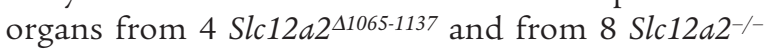
mice. Similar defects were seen in both mutant lines.

Histological examination of the cauda epididymis, where large numbers of sperm are stored in wild-type mice (Figure 4a), indicated that sperm were absent in NKCC1-deficient males. Instead, the epididymal lumen

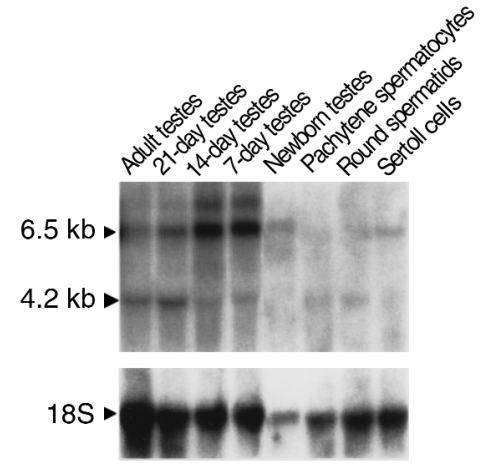

\section{Figure 6}

Northern analysis of NKCC 1 expression in the testes of maturing animals and in specific testis cell populations. A normal 6.5-kb NKCC1 transcript is observed in the testis throughout postnatal development. The presence of an alternate transcript is detected in 7- and 14-day-old mice, and this 4.2-kb transcript becomes more abundant in 21-day-old mice. This alternate transcript is also detected in pachytene spermatocytes and round spermatids in addition to the normal NKCC1 transcript, whereas only the $6.5-\mathrm{kb}$ transcript is detected in Sertoli cells. RNA loading is 20 $\mu \mathrm{g}$ for the testis samples and $10 \mu \mathrm{g}$ for the isolated cell types. Analysis of $18 \mathrm{~S}$ RNA is shown to demonstrate equal loading of the samples.

was filled with PAS-positive material and occasional cells with pyknotic nuclei (Figure 4e). Although NKCC1-deficient males (1.5-3 months old) did not have sperm in the epididymis or vas deferens, the epithelia of these tissues were indistinguishable from wild-type mice.

On gross examination, the testes of NKCC1-deficient males were smaller in size than testes of wild-type males with similar body weights. Histological analysis showed that this difference in size is related to the smaller diameter of the seminiferous tubules in these animals (compare Figures $4 \mathrm{~b}$ and $4 \mathrm{f}$ ). The morphology of the tubules was clearly abnormal in the NKCC1-deficient males, including vacuolization of the seminiferous epithelium, variable loss of germ cells, and the appearance of multinucleated germ cells in the lumen of some tubules (arrows, Figure 4f). As expected, the seminiferous tubules of mature wild-type males contain germ cells at all stages of spermatogenesis, including early spermatogonia near the periphery of each tubule, large spermatocytes in meiotic prophase, and round and elongating haploid spermatids near the lumen (Figures $4 c$ and $4 d$ ). Spermatids are easily identified by PAS-staining of the developing acrosomes (arrow, Figure 4c). Sertoli cells, the supporting cells within the seminiferous epithelium, are present in each tubule and are identified by their large, pale nuclei with characteristic central nucleoli near the basal lamina. The seminiferous tubules of NKCC1-deficient males had Sertoli cells with typical nuclear morphology (s in Figure 4h), but displayed abnormal spermatogenesis with varying degrees of germ cell loss and degeneration (Figure 4, g and h). Spermatogonia and/or early spermatocytes with darkly staining nuclei appeared to be more numerous (e in Figure 4h) than in the tubules of wild-type males (arrow, Figure 4d). Large pachytene 
spermatocytes ( $p$ in Figure 4h) were present in NKCC1deficient males, although the number was reduced in many tubules. Spermatids were absent or markedly reduced in number in all seminiferous tubules. However, small clusters of spermatids at varying stages of differentiation were seen in the mutant animals (arrows, Figure 4g). The extent of germ cell loss varied between animals in both mutant lines. In 5 animals older than 10 months, some tubules were filled with whorls of Sertoli cell cytoplasm and appeared devoid of germ cells, suggesting that the severity of the lesion may increase with advancing age.

When examined by electron microscopy, spermatids of NKCC1-deficient males displayed abnormal ultrastructural features (Figures 5b and 5c). Acrosomal defects were apparent in mutant spermatids, including abnormal placement of the acrosomal granule (arrows, Figure 5 , b and c) and vesicular structures resembling two acrosomes at opposite poles of 1 nucleus (* in Figure 5c). In contrast, wild-type spermatids at similar stages of differentiation have a central acrosomal granule within the cap-phase acrosomal vesicle (arrow, Figure 5a).

Detection of NKCC1 expression in the adult testis by Northern analysis has been reported previously (18). To define further NKCC1 expression, Northern blot analysis was carried out to compare mRNA levels during postnatal testis development (Figure 6). The typical NKCC1 transcript $(6.5 \mathrm{~kb})$ was present in testes from newborn mice, which contain only gonocytes, Sertoli cells, and interstitial cells. In testes from 7- and 14-dayold mice the $6.5-\mathrm{kb}$ transcript was predominant, although a smaller 4.2-kb NKCC1 transcript was also detected. During this period of development, gonocytes differentiate to become spermatogonia, which continue to divide and then enter meiosis during the second week of life. By 21 days old, when spermatids begin to accumulate, the ratio of the 2 NKCC1 transcripts was altered. Approximately equal levels of the 2 NKCC 1 transcripts were present by this age, and this expression pattern continued in adult mice. NKCC1 expression was also examined in pachytene spermatocytes and round spermatids isolated from adult animals and in Sertoli cells isolated from 17-day-old mice. Both NKCC1 transcripts were detected in pachytene spermatocytes and round spermatids, whereas the larger $6.5-\mathrm{kb}$ transcript was predominant in Sertoli cells.

\section{Discussion}

The two NKCC1-deficient mouse lines described here differ with respect to the mutation they carry in the Slc12a2 locus. Whereas one of the mutations likely represents a null allele, the second mouse line carries a deletion mutation in the $3^{\prime}$ cytoplasmic region. Normal levels of mRNA are detected, and it is presumed that this mRNA can be transcribed into a protein. Future verification of this depends on the identification of NKCC1 protein in these cell lines with antibodies that do not require expression of the deleted region. The mouse line carrying the deletion mutation displays a phenotype indistinguishable from the mice homozygous for the null allele. This finding is surprising because this region is not believed to be involved in binding or transport of the ions and supports an important role for the cytoplasmic region in the normal functioning of NKCC1 protein. The NKCC1 cytoplasmic tail region has been shown to be phosphorylated in response to stimuli that increase bumetanide-sensitive ion transport (36). However, none of the amino acids absent in the predicted mutant NKCC1 protein have been identified previously as targets for these regulatory modifications. We cannot rule out the possibility that the deletion of this region alters the conformation of the molecule and thereby indirectly affects phosphorylation of specific amino acids. Of the 72 amino acids absent in the deletion mutation, 55 are conserved in sharks. A number of lines of evidence suggest that ion transport in some cells is dependent on interactions of the various channels, pumps, and cotransporters with the cytoskeleton of the cell and that this interaction may be particularly important in functions such as volume regulation (37). It is therefore possible that the residues that are absent in the NKCC1-deletion mutant are required for the interaction of NKCC1 with other, as yet unidentified proteins.

The salt and water reabsorptive function of the apical NKCC2 isoform of the cotransporter in the thick ascending limb of Henle cells is well established, based on the large natriuretic effect of loop diuretics (3). Less is known about the function of NKCC1. It is expressed in the inner medullary collecting ducts where it has been suggested that it may be important in volume regulation of these cells or where it might contribute to the development and/or maintenance of the medullary osmolar gradient (7). This latter hypothesis is not supported by the studies described here, because NKCC1-deficient mice could concentrate urine in response to vasopressin. NKCC1 expression in a subpopulation of smooth muscle cells of the afferent arteriole and in the extraglomerular mesangium has led to the hypothesis that this molecule may be involved in tubuloglomerular feedback and, perhaps, renin secretion (7). Again, the failure to detect changes in blood pressure in mice placed on low- and high-salt diets suggests that other sensory mechanisms are available for modifying glomular filtration rate and renin secretion in the absence of NKCC1.

Since the preparation of this article, Flagella et al. (38) have reported the generation of Slc12a2 $2^{-/}$mice. This group reported the early death of many of the NKCC1deficient animals and a slight decrease in blood pressure of surviving animals. This difference in the phenotype may reflect environmental factors that lead to decreased survival and alteration in physiological parameters. We have bred our chimeras to B6D2 animals. Placing the mutation on a heterogeneous genetic background might have increased the size of the pups, resulting in fewer indirect physiological manifestations of loss of NKCC1 function. Alternatively, it is possible that alleles present in $\mathrm{B} 6 \mathrm{D} 2$ can compensate for loss of NKCC1 function in the kidney. Because the strain used in the studies by Flagella et al. is not described, it is not possi- 
ble to distinguish between these possibilities.

The infertility of NKCC1-deficient males is consistent with the histological analysis of the reproductive organs of these animals. Mature spermatozoa were not observed in sections of the epididymis or vas deferens. The testes of 3- to 4-day-old NKCC1-deficient mice could not be distinguished from those of wild-type littermates upon histological examination. Examination of the sections of testis from four 21-day-old NKCC1-deficient mice suggested that the first observable lesion in these animals is delayed formation of the lumen of the seminiferous tubules. By 1.5 months of age, marked abnormalities in spermatogenesis were observed in the NKCC1-deficient animals. Defects were particularly striking during the final phases of spermatogenesis, the period when haploid spermatids gradually acquire the polarized shape and unique structural features of spermatozoa. In all seminiferous tubules examined, spermatids were absent or present in very small numbers. Furthermore, spermatids present in the NKCC1-deficient males displayed abnormal ultrastructural features when examined with the electron microscope. In many seminiferous tubules the number of pachytene spermatocytes was also reduced, indicating additional abnormalities during meiotic prophase. The sections examined from 5 animals greater than 10 months of age showed extensive loss of cells, with a number of tubules containing predominantly Sertoli cells, suggesting that cellular loss may increase with advancing age.

Although the number and morphology of the Sertoli cells in the NKCC1-deficient animals is largely unchanged, altered function of these cells may lead to the progressive loss of spermatogenic cells. Sertoli cells are believed to be responsible for the formation of the specialized luminal fluid microenvironment and the secretion of fluid that transports the spermatozoa into the epididymis (39-41). This fluid has a unique ionic composition with $\mathrm{K}^{+}$concentrations 10 times higher than those seen in plasma (40). High $\mathrm{K}^{+}$levels are extremely rare in fluid secreted by epithelial tissues. However, high levels of $\mathrm{K}^{+}$are also characteristic of the endolymph of the inner ear (42). The inner ear and testicular defects observed in the NKCC1-deficient mice suggest that this cotransporter may be essential for the secretion of these high $\mathrm{K}^{+}$fluids. Salivary epithelium can also produce fluid rich in $\mathrm{K}^{+}(43)$. Whereas no alterations in the histology of this organ were observed, it is interesting to note that extremely high levels of expression of the cotransporter are detected in this tissue.

Although the composition of the luminal fluid of the seminiferous tubule has been extensively studied, a paucity of information is available concerning the secretory mechanisms underlying its formation. A model proposed by Hinton and Setchell (44) suggests that $\mathrm{K}^{+}$is pumped into the Sertoli cell from the interstitial fluid by an active process, probably one involving a $\mathrm{Na}^{+}, \mathrm{K}^{+}$translocating ATPase. This is supported by the observation that increased external potassium or decreased external $\mathrm{Na}^{+}$concentrations decrease the potential of the Sertoli cell whereas no change is observed in $\mathrm{Cl}^{-}$concentrations (44). In this model $\mathrm{K}^{+}$ moves across the adluminal surface down a concentration gradient. Additional evidence supporting this model comes from studies showing that agents that block potassium channels cause morphological defects in the seminiferous epithelium that first affect latestage spermatids (45). A possible role of NKCC1 in transport of $\mathrm{K}^{+}$at the basolateral surface of the Sertoli cell has not been extensively examined. A single report examining short circuit currents across monolayers of Sertoli cells in the presence and absence of bumetanide failed to support a role for NKCC1 in ion transport (46). Future studies comparing ion transport across Sertoli cells derived from NKCC1-deficient animals should allow us to address this question directly.

Although mouse lines carrying mutations in a number of ion channels and transporters have been identified and generated, they have provided little insight into the physiology of spermatogenesis and specifically into the production of seminal fluid. An exception is the weaver mouse that carries a mutation in Girk2, a $\mathrm{G}$ protein-coupled, inwardly rectifying $\mathrm{K}^{+}$channel (47). In the weaver mice degeneration and death of spermatogenic cells, particularly those at the most advanced stages of differentiation, have been attributed both to defects in Sertoli cells (48) and to direct effects on the germ cells (47). It is interesting to speculate that both NKCC1 and GIRK 2 are required for the production of the luminal fluid of the seminiferous tubules. Whereas under normal physiological conditions GIRK 2 promotes movement of $\mathrm{K}^{+}$into the cell, it is possible that transport of ions can occur in the reverse direction in the unique environment of the seminiferous tubule. Alternatively, it is possible that, through its association with G proteins, GIRK 2 regulates the activity of other ion transport pathways, including NKCC 1 and apical $\mathrm{K}^{+}$channels.

Our demonstration that NKCC 1 is also expressed by germ cells raises the possibility that this gene is directly required for the development and maturation of this lineage. Direct determination of the relative contribution of Sertoli cell and germ cell NKCC1 function to spermatogenesis should be possible with the development of mouse lines in which loss of NKCC1 is limited to either of these cell populations. Alternatively, approaches such as germ line transplantation of normal spermatogonia into Slc12a $2^{-/-}$animals may provide insight into this question.

Given the broad tissue distribution of NKCC1 and its proposed importance both in volume regulation and fluid secretion by epithelia, the observation of a dramatic phenotype in only the testis and inner ear is perhaps surprising. It suggests that in both secretory epithelia and in most nonepithelial cells, alternate pathways exist that, in the absence of the cotransporter, can maintain the physiological homeostasis of these organ systems and cells. Thus, in addition to the information gathered on the function of the cotransporter 
itself, these animals will facilitate the identification of new pathways important in volume regulation and secretory function of epithelia that have been difficult to measure using traditional technologies.

\section{Acknowledgments}

The authors thank B. Bagnell and V. Madden for assistance with histology and microscopy; K. Burns and T. Bartolotta for assistance with histology; M. Nguyen for assistance with molecular biology techniques; E. Hicks for assistance with animal husbandry; A. Latour for tissue culture; G. Buchold for preparing testicular RNA samples; R. Gainetdinov and M. Caron for assistance with preliminary animal behavior studies; and R. Boucher and B. Grubb for helpful discussions of the manuscript. This work was supported by National Institutes of Health grants HL-60280 (to B.H. Koller and R. Boucher) and HD-26485 (to D.A. O'Brien).

1. O'Grady, S.M., Palfrey, H.C., and Field, M. 1987. Characteristics and functions of Na-K-Cl cotransport in epithelial tissues. Am. J. Physiol. 253:C177-C192.

2. Haas, M. 1994. The Na-K-Cl cotransporters. Am. J. Physiol. 267:C869-C885.

3. Forbush, B., III, and Palfrey, H.C. 1983. $\left[{ }^{3} \mathrm{H}\right]$ bumetanide binding to membranes isolated from dog kidney outer medulla. Relationship to the $\mathrm{Na}, \mathrm{K}, \mathrm{Cl}-$ cotransport system. J. Biol. Chem. 258:11787-11792.

4. Payne, J.A., and Forbush, B., III. 1994. Alternatively spliced isoforms of the putative renal $\mathrm{Na}-\mathrm{K}-\mathrm{Cl}$ cotransporter are differentially distributed within the rabbit kidney. Proc. Natl. Acad. Sci. USA. 91:4544-4548.

5. Ginns, S.M., et al. 1996. Immunolocalization of the secretory isoform of $\mathrm{Na}$ $\mathrm{K}-\mathrm{Cl}$ cotransporter in rat renal intercalated cells. J. Am. Soc. Nephrol. 7:2533-2542.

6. Kaplan, M.R, et al. 1996. Apical localization of the Na-K-Cl cotransporter, rBSC1, on rat thick ascending limbs. Kidney Int. 49:40-47.

7. Kaplan, M.R., Plotkin, M.D., Brown, D., Hebert, S.C., and Delpire, E. 1996. Expression of the mouse $\mathrm{Na}-\mathrm{K}-2 \mathrm{Cl}$ cotransporter, $\mathrm{mBSC} 2$, in the terminal inner medullary collecting duct, the glomerular and extraglomerular mesangium, and the glomerular afferent arteriole. J. Clin. Invest. 98:723-730.

8. Palfrey, H.C., Feit, P.W., and Greengard, P. 1980. cAMP-stimulated cation cotransport in avian erythrocytes: inhibition by "loop" diuretics. Am. J. Physiol. 238:C139-C148.

9. Palfrey, H.C., Silva, P., and Epstein, F.H. 1984. Sensitivity of cAMP-stimulated salt secretion in shark rectal gland to "loop" diuretics. Am. J. Physiol. 246:C242-C246.

10. Lytle, C.Y., Xu, J.-C., Biemesderfer, D., Haas, M., and Forbush, B., III. 1992. The Na-K-Cl cotransport protein of shark rectal gland. J. Biol. Chem. 267:25428-25437.

11. Takeuchi, S., Marcus, D.C., and Wangemann, P. 1992. Ca+-activated nonselective cation, maxi $\mathrm{K}^{+}$and $\mathrm{Cl}^{-}$channels in apical membrane of marginal cells of stria vascularis. Hear. Res. 84:86-96.

12. Sun, A., Saltzberg, S.N., Kikeri, D., and Hebert, S.C. 1990. Mechanisms of cell volume regulation by the mouse medullary thick ascending limb of Henle. Kidney Int. 38:1019-1029.

13. Paulais, M., Valdez, I.H., Fox, P.C., Evans, R.L., and Turner, R.J. 1996. Ion transport systems in human labial acinar cells. Am. J. Physiol. 270:g213-g219.

14. Crouch, J.J., Sakaguchi, N., Lytle, C., and Schulte, B.A. 1997. Immunohistochemical localization of the Na-K-Cl co-transporter (NKCC1) in the gerbil inner ear. J. Histochem. Cytochem. 45:773-778.

15. Goto, S., Oshima, T., Ikeda, K., Ueda, N., and Takasaka, T. 1997. Expression and localization of the $\mathrm{Na}-\mathrm{K}-2 \mathrm{Cl}$ cotransporter in the rat cochlea. Brain Res. 765:324-326

16. Haas, M., and Forbush, B., III. 1986. $\left[{ }^{3} \mathrm{H}\right]$ bumetanide binding to duck red cells. Correlation with inhibition of $(\mathrm{Na}+\mathrm{K}+2 \mathrm{Cl})$ cotransport. J. Biol. Chem. 261:8434-8441.

17. Kracke, G.R., and Dunham, P.B. 1987. Effect of membrane potential on furosemide inhibitable sodium influxes in human red blood cells. J. Membr. Biol. 98:117-124.

18. Delpire, E., Rauchman, M.I., Beier, D.R., Hebert, S.C., and Gullans, S.R. 1994 Molecular cloning and chromosome localization of a putative basolateral $\mathrm{Na}^{+}-\mathrm{K}^{+}-2 \mathrm{Cl}^{-}$cotransporter from mouse inner medullary collecting duct (mIMCD-3) cells. J. Biol. Chem. 269:25677-25683.

19. Tuck, R.R., Setchell, B.P., Waites, G.M., and Young, J.A. 1970. The composition of fluid collected by micropuncture and catheterization from the seminiferous tubules and rete testis of rats. Pflugers Arch. 318:225-243.
20. Dombrowitz, D., Flammand, V., Brigman, K.K., Koller, B.H., and Kinet, J.-P. 1993. Abolition of anaphylaxis by targeted disruption of the high affinity immunoglobulin e receptor $\alpha$ chain gene. Cell. 75:969-976.

21. Mohn, A., and Koller, B.H. 1995. Genetic manipulation of embryonic stem cells. In DNA cloning 4. D.M. Glover and B.D. Hames, editors. Oxford University Press. New York, NY. 143-184.

22. Miller, S.A., Dykes, D.D., and Polesky, H.F. 1988. A simple salting out procedure for extracting DNA from human nucleated cells. Nucleic Acids Res. 16:1215.

23. O’Brien, D.A., Gabel, C.A., Rockett, D.L., and Eddy, E.M. 1989. Receptormediated endocytosis and differential synthesis of mannose 6-phosphate receptors in isolated spermatogenic and sertoli cells. Endocrinology. 125:2973-2984.

24. O'Brien, D.A. 1993. Isolation, separation, and short-term culture of spermatogenic cells. In Methods in toxicology.Volume 3A. R.E. Chapin and J.J. Heindel, editors. Academic Press. San Diego, CA. 246-263.

25. Kroczek, R.A., and Siebert, E. 1990. Optimization of northern analysis by vacuum-blotting, RNA-transfer visualization, and ultraviolet fixation. Anal. Biochem. 184:90-95.

26. Krege, J.H., Hodgin, J.B., Hagaman, J.R., and Smithies, O. 1995. A computerized system for measuring blood pressure in mice. Hypertension. 25:1111-1115.

27. Ito, M., et al. 1995. Regulation of blood pressure by the type IA receptor for angiotensin II. Proc. Natl. Acad. Sci. USA. 92:3521-3525.

28. Xu, J.-C., et al. 1994. Molecular cloning and functional expression of the bumetanide-sensitive Na-K-Cl cotransporter. Proc. Natl. Acad. Sci. USA 91:2201-2205.

29. Fuller, J.L., and Wimer, R.E. 1966. Neural, sensory, and motor functions. In Biology of the laboratory mouse. E.L. Green, editor. Dover Publications Inc. New York, NY. 609-628.

30. Probst, F.J., et al. 1998. Correction of deafness in shaker-2 mice by an unconventional myosin in a BAC transgene. Science. 280:1444-1447.

31. Wang, W., Van de Water, T., and Lufkin, T. 1998. Inner ear and maternal reproductive defects in mice lacking the $H m \times 3$ homeobox gene. Development. 125:621-634.

32. Erkman, L., et al. 1996. Role of transcription factors Brn-3.1 and Brn-3.2 in auditory and visual system development. Nature. 381:603-606.

33. Delpire, E., Lu, J., England, R., Dull, C., and Thorne, T. 1999. Deafness and imbalance associated with inactivation of the secretory Na-K-2Cl co-transporter. Nat. Genet. 22:192-195.

34. Dixon, M.J., et al. 1999. Mutation of the Na-K-Cl co-transporter gene Slc12a2 results in deafness in mice. Hum. Mol. Genet. 8:1579-1584.

35. Plotkin, M.D., et al. 1997. Expression of the $\mathrm{Na}^{+}-\mathrm{K}^{+}-2 \mathrm{Cl}^{-}$cotransporter BSC2 in the nervous system. Am. J. Physiol. 272:C173-C183.

36. Lytle, C., and Forbush, B., III. 1992. The Na-K-Cl cotransporter protein of shark rectal gland. J. Biol. Chem. 267:25438-25443.

37. Matthews, J.B., Smith, J.A., Mun, E.C., and Sicklick, J.K. 1998. Osmotic regulation of intestinal epithelial $\mathrm{Na}^{+}-\mathrm{K}^{+}-\mathrm{Cl}^{-}$cotransport: role of $\mathrm{Cl}^{-}$and $\mathrm{F}$-actin Am. J. Physiol. 274:C697-C706.

38. Flagella, M., et al. 1999. Mice lacking the basolateral Na-K-2Cl cotransporter have impaired epithelial chloride secretion and are profoundly deaf.J. Biol. Chem. 274:26946-26955.

39. Cheung, Y.M., Hwang, J.C., and Wong, P.Y.D. 1977. In vitro measurement of rate of fluid secretion in rat isolated seminiferous tubules: effects of metabolic inhibitors and ions. J. Physiol. (Lond.). 269:1-15.

40. Levine, N., and Marsh, D.J. 1971. Micropuncture studies of the electrochemical aspects of fluid and electrolyte transport in individual seminiferous tubules, the epididymis and the vas deferens in rats. J. Physiol. (Lond.). 213:557-570.

41. Setchell, B.P. 1970. The secretion of fluid by the testes of rats, rams and goats, with some observations on the effects of age, cryptorchidism and hypophysectomy. J. Reprod. Fertil. 23:79-85.

42. Johnstone, C.G., Schmidt, R.S., and Johnstone, B.M. 1963. Sodium and potassium in vertebrate cochlear endolymph as determined by flame microspectrophotometry. Comp. Biochem. Physiol. 9:335-341.

43. Paulais, M., Cragoe, E.J., Jr., and Turner, R.J. 1994. Ion transport mechanisms in rat parotid intralobular striated ducts. Am. J. Physiol. 266:c1594-c1602

44. Hinton, B.T., and Setchell, B.P. 1993. Fluid secretion and movement. In The Sertolicell. L.D. Russell and M.D. Griswold, editors. Cache River Press. Clearwater, FL. 249-267.

45. Setchell, B.P., Ploen, L., and Ritzen, E.M. 1998. Reduction in fluid secretion by rat testis by drugs that block potassium channels. J. Reprod. Fertil. 112:87-94.

46. Ko, W.H., Chan, H.C., Chew, S.B., and Wong, P.Y.D. 1998. Regulated anion secretion in cultured epithelia from sertoli cells of immature rats. J. Physiol. (Lond.). 2:471-480.

47. Harrison, S.M., and Roffler-Tarlov, S.K. 1998. Cell death during development of testis and cerebellum in the mutant mouse weaver. Dev. Biol. 195:174-186.

48. Verina, T., et al. 1995. Degeneration of sertoli and spermatogenic cells in homozygous and heterozygous weaver mice. J. Neurogenet. 9:251-265. 